\title{
Analbuminämie in Kombination mit Lipödem bei zwei Geschwistern
}

\author{
Analbuminaemia in Combination with Lipoedema in Two Siblings
}

Autoren

Institute
W. Schmeller ${ }^{1}$, D. Hartwig ${ }^{2}$

${ }^{1}$ Hanse-Klinik, Fachklinik für Liposuktion und operativ-ästhetische Dermatologie, Lübeck

${ }^{2}$ Laborärztliche Gemeinschaftspraxis, Lübeck
Bibliografie

DOI http://dx.doi.org/

$10.1055 / \mathrm{s}-0032-1306797$

Online-Publikation: 3.4.2012

Akt Dermatol 2012; 38: 177-182

(c) Georg Thieme Verlag KG

Stuttgart · New York

ISSN 0340-2541

Korrespondenzadresse

Prof. Dr. med.

Wilfried Schmeller

Hanse-Klinik

St.-Jürgen-Ring 66

23564 Lübeck

ws@hanse-klinik.com

\section{Zusammenfassung \\ $\nabla$}

Vorgestellt werden zwei türkische Schwestern mit dem seltenen Krankheitsbild der kongenitalen Analbuminämie in Kombination mit einem Lipödem. Während die Analbuminämie, die immer mit einer Hyperlipidämie und einer erhöhten Blutsenkungsgeschwindigkeit (BSG) einhergeht, subjektiv kaum Beschwerden bereitet und des-

\section{Einleitung \\ $\nabla$}

Die angeborene Analbuminämie ist eine ausgesprochen seltene Erkrankung. Seit der Erstbeschreibung 1954 [2] wurden bisher (Februar 2012) lediglich 50 Fälle in der Weltliteratur beschrieben [17]; es wird eine Inzidenz von < 1: 1 Million vermutet [5]. Neben Beschreibungen aus europäischen Ländern existieren Kasuistiken aus Nord- und Südafrika, USA und Kanada, Russland, Irak, Israel und Japan $[3,7,11,12,16,17]$.

Klinisch ist die Erkrankung durch Müdigkeit, Ödeme und niedrigen Blutdruck gekennzeichnet. Da diese Symptome aber häufig nur diskret ausgeprägt sind, wird die Diagnose meist per Zufall durch die Serum-Eiweiß-Elektrophorese gestellt. Dabei ist das hervorstechende Merkmal die ausgeprägte Verminderung des Albumins. Zusätzlich finden sich eine stark erhöhte Blutsenkungsgeschwindigkeit (BSG) sowie eine Hyperlipidämie mit erhöhten Werten von LDL-Cholesterin und meist auch Triglyzeriden.

Die Analbuminämie, die als Neumutation entstehen kann oder autosomal rezessiv vererbt wird, ist Folge von Defekten des Albumingens auf dem Chromosom 4, Genlocus q13.3 [5,7]. Während bei homozygoten Individuen allenfalls noch minimale Albuminmengen nachweisbar sind, sind bei heterozygoten Trägern nur geringfügige Verminderungen der physiologischen Albuminkonzentration $(35-55 \mathrm{~g} / \mathrm{l})$ nachweisbar. halb häufig lange unentdeckt bleibt, ist das Lipödem aufgrund der unproportionierten Unterhautfettvermehrung mit Ödemen, Druckschmerzen und Hämatomen klinisch gut erkennbar.

Die Analbuminämie benötigt aufgrund fehlender Symptome keine spezifische Therapie; beim Lipödem lassen sich Morphologie und Beschwerden durch Liposuktion deutlich bessern. Die Kombination beider Erkrankungen sollte bekannt sein.

Männer und Frauen sind von der Analbuminämie gleich häufig betroffen. Beim weiblichen Geschlecht wird bei einem Teil der Fälle eine Lipodystrophie beschrieben. Dies ist ein Überbegriff für eine umschriebene, unproportionierte Unterhautfettverminderung (Lipoatrophie) oder -vermehrung (Lipohypertrophie). Tritt letztere in der unteren Körperhälfte („lower limb obesity“) in Kombination mit Ödemen, Schmerzen und Hämatomen auf, handelt es sich um das Krankheitsbild des Lipödems.

Das Lipödem ist eine nur bei Frauen auftretende, chronisch progrediente, umschriebene und z.T. disfigurierende Unterhautfettvermehrung unklarer Ätiologie. Krankheitstypisch sind ödembedingte Spannungs-, Berührungs- und Druckschmerzen sowie eine auffallende Hämatomneigung $[19,22,24]$.

Diese 1940 erstmals beschriebene Erkrankung [1], bei der eine autosomal dominante Vererbung vermutet wird [6], war als eigene Entität lange umstritten [9]. Bis Ende des 20.Jahrhunderts konnte nur ein Teilaspekt des Lipödems durch konservative Maßnahmen behandelt werden; mit manueller Lymphdrainage und anschließender Kompression (kombinierte physikalische Entstauung, KPE) ließen sich lediglich die Ödeme und die dadurch erzeugten Beschwerden vermindern. Seit etwa einem Jahrzehnt ist es nun auch möglich, das krankhaft vermehrte Unterhautfettgewebe operativ mittels Liposuktion $\mathrm{zu}$ 
reduzieren; beide Behandlungsverfahren sind Bestandteil der aktuellen Leitlinien zum Lipödem $[21,24]$.

\section{Fallberichte}

Patientin 1 (N. F., 42 Jahre)

Anamnese

Bei der 42-jährigen türkischen Patientin war im 25.Lebensjahr im Rahmen einer Durchuntersuchung eine Analbuminämie festgestellt worden. Sie litt unter ständiger Müdigkeit und Beinödemen. Nach der Schwangerschaft im Alter von 38 Jahren erfolgte eine „dramatische“ Zunahme der Beinumfänge bei gleichzeitiger Gewichtsvermehrung von etwa 70 auf $90 \mathrm{~kg}$. Damit einhergehend verstärktes Auftreten vorwiegend abendlicher Schwellungen mit Spannungsgefühl, ausgeprägter Druckempfindlichkeit und Neigung zu Blutergüssen. Eine vom 21. bis 25 . Lebensjahr durchgeführte konservative Therapie mit manuellen Lymphdrainagen $2 \times$ pro Woche und Tragen von rundgestrickten Kompressionsstrümpfen bewirkte keine deutliche Befund- bzw. Beschwerdebesserung.

\section{Familienanamnese}

Von den insgesamt 5 Kindern der Familie ist außer bei ihr (Pat. 1) auch bei der 3 Jahre jüngeren Schwester (Pat. 2, E.M.) eine Analbuminämie bekannt (s. u.). Eine weitere Schwester (M. B., 23 Jahre) konnte ebenfalls von uns untersucht werden. Bei ihr zeigte sich eine unauffällige Serum-Elektrophorese ( $\bullet$ Abb. 1, unten); damit konnte eine Analbuminämie sicher ausgeschlossen werden. Bei der Mutter besteht eine Hypercholesterinämie; ob zusätzlich eine Albuminverminderung vorliegt, war nicht eruierbar. Bei dem 63-jährigen Vater und der 60-jährigen Mutter sind keine gesundheitlichen Probleme, insbesondere keine Herzerkrankungen, bekannt.

\section{Befund}

Bei einer Größe von $154 \mathrm{~cm}$ und einem Gewicht von $91,6 \mathrm{~kg}$ (BMI $38,4 \mathrm{~kg} / \mathrm{m}^{2}=$ Adipositas Grad 2) fand sich bei schlankem Rumpf eine ausgeprägte Disproportion zum voluminösen Unterkörper $(\bullet$ Abb.2a). Es bestanden sowohl an Hüften und Gesäß als auch an Ober- und Unterschenkeln deutliche Vermehrungen des Unterhautfettgewebes von relativ fester Konsistenz mit überhängenden subkutanen Gewebeanteilen in der Knöchelregion medial, lateral und ventral.

Palpatorisch Knotenbildung subkutan, Druckschmerz an Oberund Unterschenkeln, vereinzelt Hämatome, eindrückbare Ödeme prätibial. Mäßig verdickte Hautfältelung an den Zehen bei unauffälliger Fußform, keine Dellbarkeit der Fußrücken; das Stemmer'sche Zeichen war negativ, die Bisgaard'schen Kulissen waren fettbedingt verstrichen. Auch an den Ober- und Unterarmen Vermehrung von Unterhautvolumen mit Stufenbildung in der Ellbogenregion.

\section{Laborbefunde}

Besonders auffällig war - bei mäßig erniedrigtem Gesamteiweiß - die Elektrophorese mit ausgeprägter Erniedrigung des Albumins bei Vermehrung der Globuline ( $\bullet$ Abb. 1, oben). Weitere relevante Laborbefunde sind in $\bullet$ Tab. 1 dargestellt; dabei zeigte sich eine erhöhte BSG bei unauffälligem CRP, eine starke Erhöhung von Cholesterin im Serum sowie die vermehrte Ausscheidung des alpha-1-Mikroglobulins im Urin. Erhöht waren

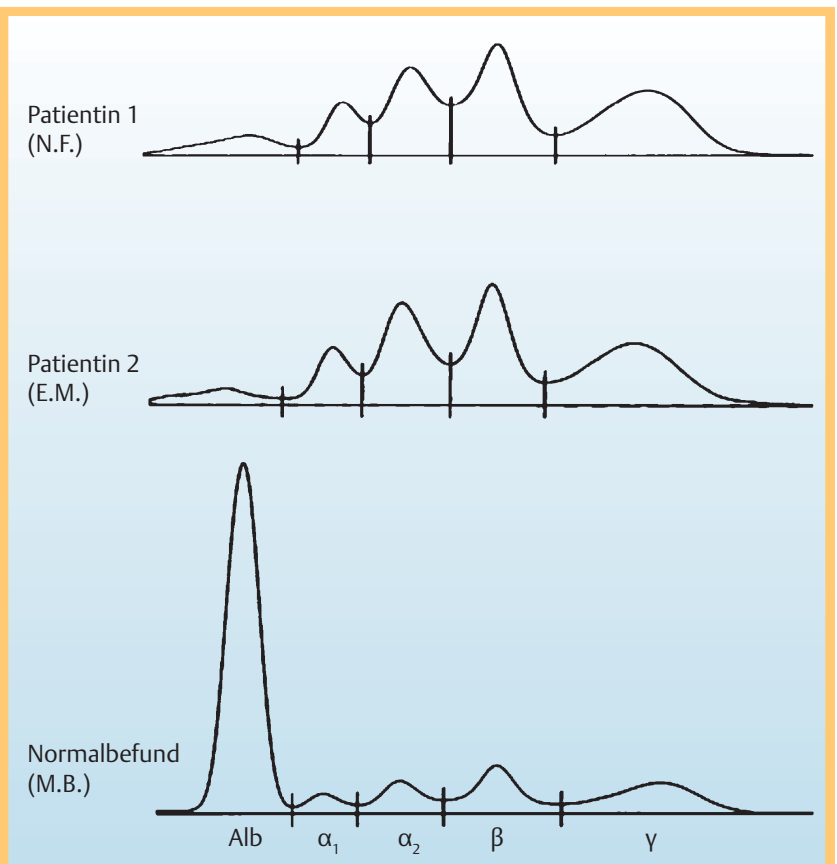

Abb. 1 Elektrophoresen von Pat. 1 und Pat. 2 (Analbuminämie) im Vergleich mit der gesunden Schwester (Normalbefund).

ferner alpha-1-Antitrypsin, Transferrin, Fibrinogen, thyroxinbindendes Globulin (TbG) und Cholinesterase.

\section{Therapie}

Über einen Zeitraum von 9 Monaten (Juli 2010 bis März 2011) wurden die sechs in $\bullet$ Tab. 2 aufgeführten Liposuktionen durchgeführt; dabei wurden insgesamt $22400 \mathrm{ml}$ Fettgewebe entfernt. Alle operativen Eingriffe wurden im Rahmen der Abrechnungsbestimmungen zur Hessischen Beihilfeverordnung (HBeihVO) als beihilfefähig anerkannt. Neben einer ausgeprägten Formverbesserung ( $\bullet$ Abb. $\mathbf{2 b}$ zeigt den Befund nach 4 Sitzungen) wurde auch bezüglich Müdigkeit in den Beinen, Schwere- und Spannungsgefühl sowie Schwellneigung eine deutliche Besserung angegeben.

\section{Patientin 2 (E. M., 39 Jahre)}

Anamnese

Bei der 3 Jahre jüngeren Schwester bestanden ebenfalls seit der Pubertät "dicke Beine“; auch bei ihr kam es während der ersten Schwangerschaft im Alter von 34 Jahren zu einer deutlichen Zunahme der Beinumfänge bei einer Gewichtsvermehrung von 62 auf $84 \mathrm{~kg}$. Trotz postpartaler Reduktion auf $77 \mathrm{~kg}$ zeigte sich kaum eine Umfangsreduktion an den Beinen; zusätzlich kam es zum Auftreten von Beschwerden mit Beinschwellungen, Druckempfindlichkeit der Unterschenkel und Neigung zu Blutergüssen. Seit über 20 Jahren wurden Kompressionsstrumpfhosen (früher rund-, jetzt flachgestrickt, KKL II) getragen, die eine weitgehende, aber nicht vollständige Schmerzfreiheit bewirkten. Manuelle Lymphdrainagen wurden zuletzt vor 15 Jahren durchgeführt.

\section{Befund}

Bei einer Größe von $151 \mathrm{~cm}$ und einem Gewicht von $74,4 \mathrm{~kg}$ (BMI $32,5 \mathrm{~kg} / \mathrm{m}^{2}=$ Adipositas Grad 1) fand sich morphologisch eben- 

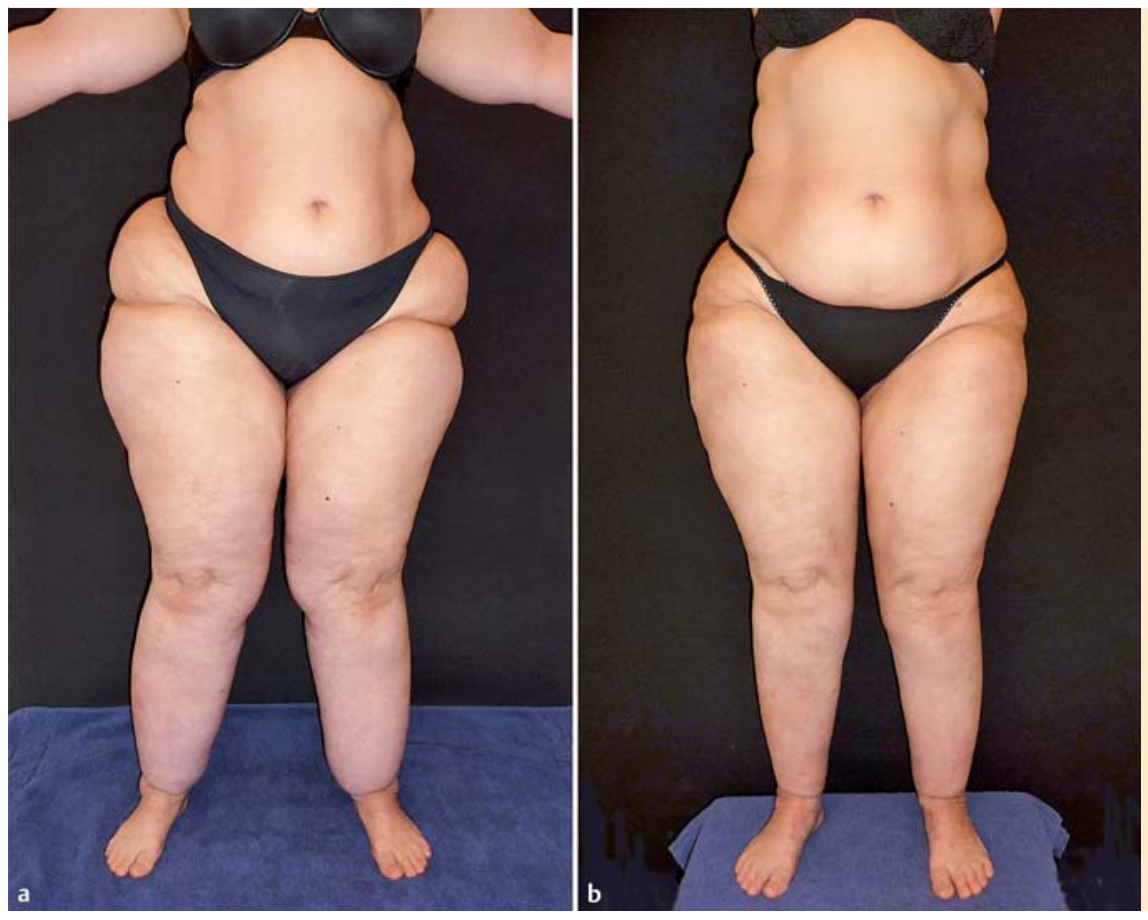

Abb.2 a Pat. 1. Analbuminämie mit Lipödem, präoperativ. b Pat. 1. Z. n. 4 Liposuktionen mit Entfernung von $18300 \mathrm{ml}$ Fettgewebe.

falls eine Disproportion mit schlankem Ober- und voluminösem Unterkörper ( $\bullet$ Abb.3a). Die subkutan gelegene Umfangsvermehrung war besonders an den Unterschenkeln ausgeprägt ( Abb.3c).

\section{Laborwerte}

Zusätzlich zu den bei der Schwester bereits aufgeführten Veränderungen waren auch noch die Triglyzeride deutlich erhöht (๑ Tab. 1).

\section{Therapie}

In der ersten Sitzung Entfernung von $4300 \mathrm{ml}$ Fettgewebe aus den Unterschenkeln ( $\mathbf{A b b} \mathbf{3} \mathbf{3}$ und $\bullet$ Abb.3d). Dadurch beidseitige Umfangsverminderung in Wadenmitte von $9 \mathrm{~cm}$ mit Besserung der Beschwerden. Die Kosten des Eingriffs wurden ebenfalls von der Krankenkasse (IKK) übernommen.

\section{Besprechung \\ $\nabla$}

Von einer angeborenen Analbuminämie wird gesprochen, wenn die mittels Immunoassay bestimmte Serum-Albumin-Konzentration unter $1 \mathrm{~g} / \mathrm{l}$ liegt $[18,20]$; in diesen Fällen ist in der Serum-Eiweiß-Elektrophorese die Albuminbande nahezu verschwunden.

Da Albumine für 80 Prozent des kolloid-osmotischen Drucks des Plasmas verantwortlich sind, wären bei einer Analbuminämie eine ausgeprägte Hypotonie und eine starke Ödemneigung zu erwarten.

Die kongenitale Verminderung der Albumine wird vom Körper durch eine vermehrte Synthese von Nicht-Albumin-Proteinen kompensiert; zu diesen gehören z.B. - neben Coeruloplasmin und thyroxinbindendem Globulin - positive Akut-Phase-Proteine (alpha-1-Antitrypsin, C3, Fibrinogen) und ein negatives Akut-Phase-Protein (Transferrin). Deren Vermehrung erhöht nicht nur den kolloid-osmotischen Druck, sondern hält ferner wohl auch die Transportfunktion der Eiweiße aufrecht. Zusätz- lich könnten Kompensationsmechanismen des Kreislaufs (Druckänderung in den Kapillaren) an der Aufrechterhaltung eines weitgehend normalen Blutdrucks beteiligt sein.

Während die erworbene Hypalbuminämie bei schweren Erkrankungen, wie z. B. dem nephrotischen Syndrom, mit einer schlechten Prognose korreliert ist [20], sind bei Patienten mit angeborener Analbuminämie die klinischen Erscheinungen der Proteinverminderung immer „erstaunlich unscheinbar“ [16]; die Diagnose wird daher meist zufällig und meist erst im Erwachsenenalter (Durchschnittsalter 24 Jahre) gestellt [12,20].

Ein unseres Wissens bisher nicht beschriebener Befund war die Erhöhung des alpha-1-Mikroglobulins im Urin. Diese ist meist Folge einer Schädigung des Tubulusapparates der Nieren. Da sich im vorliegenden Fall jedoch kein Anhalt für einen Nierenschaden fand, könnte auch eine erhöhte Expression des alpha-1-Mikroglobulins aufgrund der Analbuminämie vermutet werden. Normalerweise wird dieses Protein glomerulär filtriert und anschließend in den Nierentubuli rückresorbiert. Bei erhöhtem Anfall wäre eine Überschreitung der Rückresorptionskapazität denkbar.

Der Grund für die auffallend erhöhte Blutsenkungsreaktion ist die Änderung des Verhältnisses zwischen dem reduzierten Albumin und den erhöhten Globulinen [12]. Die normalen Werte der CRP demonstrieren, dass es sich nicht um eine entzündlich bedingte Reaktion handelt.

Ob die „up-regulation“ der Nicht-Albumin-Proteine auch die Erhöhung des Cholesterins (und vereinzelt der Triglizeride) verursacht, ist nicht bekannt; diesbezüglich wurde auch eine reduzierte intravaskuläre Lipolyse als Folge der Albuminverminderung diskutiert [10]. Ebenso ist unklar, ob die verstärkte Lipogenese pathophysiologisch entscheidend für das vermehrte Subkutanvolumen des Lipödems bei den betroffenen Frauen ist. Bezüglich der Therapie der immer vorhandenen Hypercholesterinämie (mit der potenziellen Spätfolge in Form der Arteriosklerose) gibt es keine klaren Empfehlungen. Ob aufgrund der Erhöhung von LDL-Cholesterin - u.U. zusammen mit Triglyzeriden - eine Dauerbehandlung mit Lipidsenkern in Form einer 


\begin{tabular}{|c|c|c|c|c|}
\hline Untersuchung & Patientin 1 & Patientin 2 & Referenzbereich & Einheit \\
\hline BSG (1 h) & 62 & 78 & $0-13$ & $\mathrm{~mm}$ \\
\hline \multicolumn{5}{|l|}{ Serumproteine/Metabolite } \\
\hline alpha-1-Antitrypsin & 3,60 & 3,70 & $0,9-2,0$ & $\mathrm{~g} / \mathrm{l}$ \\
\hline Transferrin & 5,70 & 6,20 & $2,3-4,3$ & $g / 1$ \\
\hline alpha-2-Coeruloplasmin & 0,59 & 0,66 & $0,2-0,6$ & $\mathrm{~g} / \mathrm{l}$ \\
\hline $\lg G$ & 1708,00 & 1730,00 & $564-1765$ & $\mathrm{mg} / \mathrm{dl}$ \\
\hline $\lg A$ & 154,00 & 294,00 & $85-385$ & $\mathrm{mg} / \mathrm{dl}$ \\
\hline $\lg M$ & 192,00 & 239,00 & $45-250$ & $\mathrm{mg} / \mathrm{dl}$ \\
\hline Kreatinin & 0,70 & 0,80 & $0,4-1,2$ & $\mathrm{mg} / \mathrm{dl}$ \\
\hline CRP & 3,00 & 3,30 & $<6,0$ & $\mathrm{mg} / \mathrm{l}$ \\
\hline C3c-Komplement & 1,50 & 2,00 & $0,9-1,8$ & $\mathrm{~g} / \mathrm{l}$ \\
\hline Fibrinogen & 5,50 & 6,30 & $2,4-5,0$ & $g / 1$ \\
\hline Thyroxine-Binding-Globulin & 43,80 & 49,10 & $11,3-28,9$ & $\mathrm{mg} / \mathrm{l}$ \\
\hline Albumin & $<0,35$ & $<0,35$ & $35-55$ & $g / 1$ \\
\hline Gesamteiweiß & 5,10 & 5,60 & $6,6-8,7$ & $\mathrm{~g} / \mathrm{dl}$ \\
\hline \multicolumn{5}{|l|}{ Serumelektrophorese } \\
\hline Albumin & 8,00 & 6,10 & $59-70,6$ & $\%$ \\
\hline alpha-1-Globulin & 10,00 & 10,60 & $2,1-4,4$ & $\%$ \\
\hline alpha-2-Globulin & 22,00 & 25,00 & $5,2-9,7$ & $\%$ \\
\hline beta-Globulin & 28,50 & 26,90 & $7,3-12,2$ & $\%$ \\
\hline gamma-Globulin & 31,50 & 31,40 & $11,2-19,9$ & $\%$ \\
\hline \multicolumn{5}{|l|}{ Enzyme } \\
\hline GPT & 19,00 & 16,00 & $<34$ & $\mathrm{U} / \mathrm{I}$ \\
\hline GOT & 27,00 & 25,00 & $<31$ & $\mathrm{U} / \mathrm{I}$ \\
\hline Cholinesterase & 15110,00 & 13985,00 & $4650-10440$ & $U / I$ \\
\hline \multicolumn{5}{|l|}{ Lipoproteine } \\
\hline Cholesterin & 491,00 & 488,00 & $130-200$ & $\mathrm{mg} / \mathrm{dl}$ \\
\hline Triglyzeride & 102,00 & 253,00 & $40-150$ & $\mathrm{mg} / \mathrm{dl}$ \\
\hline HDL-Cholesterin & 107,00 & 85,00 & $>35$ & $\mathrm{mg} / \mathrm{dl}$ \\
\hline LDL-Cholesterin & 364,00 & 352,00 & $<155$ & $\mathrm{mg} / \mathrm{dl}$ \\
\hline \multicolumn{5}{|l|}{ Elektrolyte } \\
\hline Kalium & 4,20 & 4,80 & $3,6-5,2$ & $\mathrm{mmol} / \mathrm{l}$ \\
\hline Natrium & 142,00 & 141,00 & $135-150$ & $\mathrm{mmol} / \mathrm{l}$ \\
\hline Kalzium & 2,19 & 2,15 & $2,1-2,6$ & $\mathrm{mmol} / \mathrm{l}$ \\
\hline \multicolumn{5}{|l|}{ Gerinnung } \\
\hline Quick & 94,00 & 120,00 & $70-120$ & $\%$ \\
\hline INR & 1,03 & 0,90 & $0,9-1,2$ & \\
\hline PTT & 38,00 & 32,00 & $20-40$ & sec \\
\hline \multicolumn{5}{|l|}{ Urinproteine } \\
\hline Gesamteiweiß & $<50$ & $<50$ & $<70$ & $\mathrm{mg} / \mathrm{l}$ \\
\hline $\lg G$ & $<3$ & 5,00 & $<10$ & $\mathrm{mg} / \mathrm{g}$ Krea \\
\hline Albumin & $<10$ & $<10$ & $<20$ & $\mathrm{mg} / \mathrm{l}$ \\
\hline alpha-1-Mikroglobulin & 23,50 & 57,70 & $<12$ & $\mathrm{mg} / \mathrm{l}$ \\
\hline alpha-1-Mikroglobulin & 36,32 & 24,93 & $<14$ & $\mathrm{mg} / \mathrm{g}$ Krea \\
\hline alpha-2-Makroglobulin & $<2,4$ & $<2,4$ & $<6$ & $\mathrm{mg} / \mathrm{l}$ \\
\hline beta-2-Mikroglobulin & $<0,2$ & $<0,2$ & $<2$ & $\mathrm{mg} / \mathrm{l}$ \\
\hline
\end{tabular}

ab. 1 Analbuminämie. Relevante Laborwerte.
Primärprophylaxe sinnvoll ist, wird in der Literatur nicht eindeutig beantwortet [4]. Einerseits wurde eine Therapie mit Statinen empfohlen [16] bzw. nach deren Einsatz eine Normalisierung der Cholesterin- und Triglyzeridwerte beschrieben [12]; andererseits wurde erwähnt, dass die bestehende Hyperlipidämie wohl nicht zu einer Arteriosklerose führt [20]. So wurden bei Nachuntersuchungen der ersten zwei in der Literatur beschriebenen Patientinnen über den Zeitraum von 1953 bis 1992 trotz kontinuierlich hoher Cholesterinwerte keine Hinweise für eine Arteriosklerose bzw. für thrombotische Ereignisse gefunden [15]. Demgegenüber berichtet eine Kasuistik über einen Mann mit Analbuminämie, bei dem bereits im 37. Lebensjahr aufgrund einer ausgeprägten koronaren Herzkrankeit eine Bypassoperation notwendig war [8].
Tab. 2 Fettgewebsreduktion durch Liposuktion (pro Sitzung).

\section{Patientin 1}

1. Hüften und Oberschenkel außen

$6500 \mathrm{ml}$

2. Oberschenkel innen und Knie innen

$4200 \mathrm{ml}$

3. Unterschenkel innen und außen

$3700 \mathrm{ml}$

4. Gesäß

$3900 \mathrm{ml}$

5. Ober- und Unterarme

$1700 \mathrm{ml}$

6. Oberschenkel vorne und hinten

$2400 \mathrm{ml}$

$22400 \mathrm{ml}$

Patientin 2

1. Unterschenkel innen und außen

$4300 \mathrm{ml}$ 

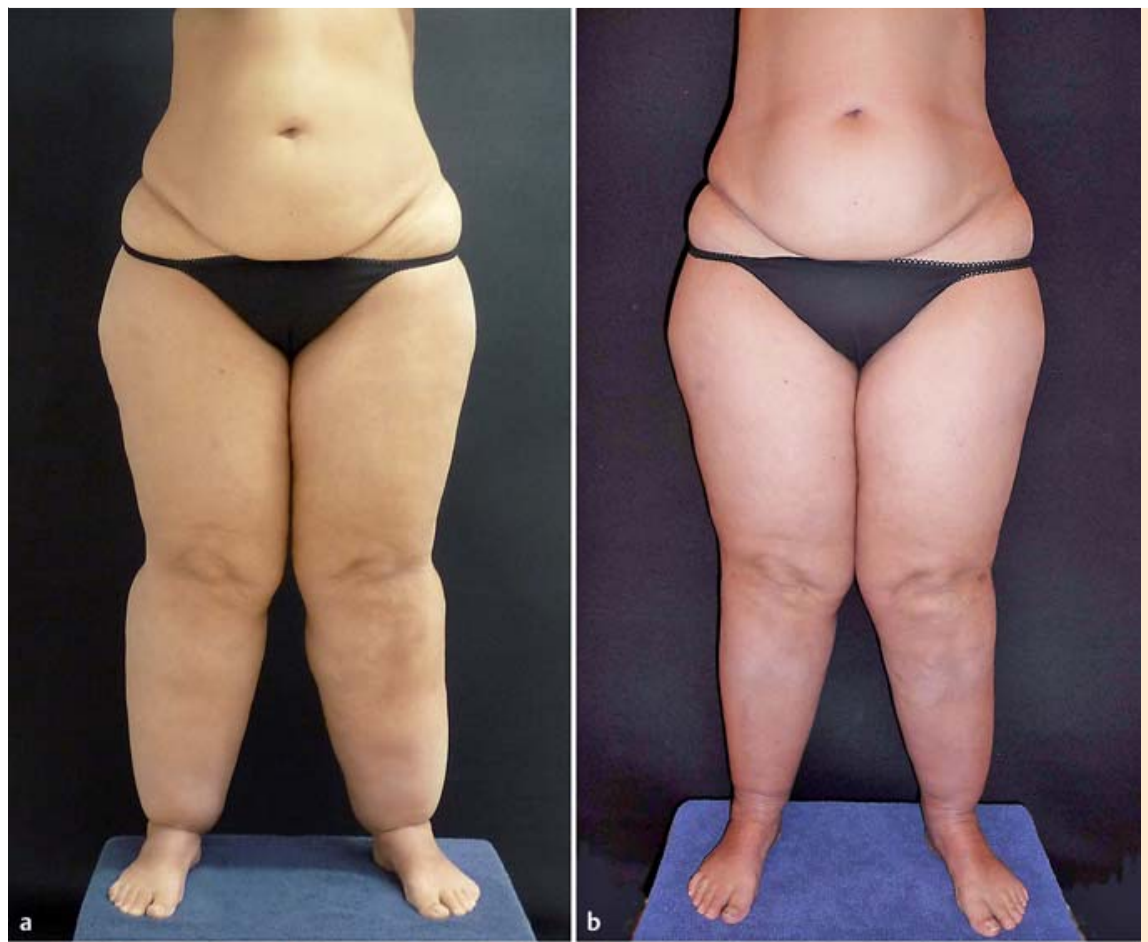

Abb.3 a Pat. 2. Analbuminämie mit Lipödem, präoperativ. b Pat. 2. Z. n. Liposuktion der Unterschenkel. c Pat. 2. Unterschenkel präoperativ. d Pat. 2. Z.n. Entfernung von $4300 \mathrm{ml}$ Fettgewebe.
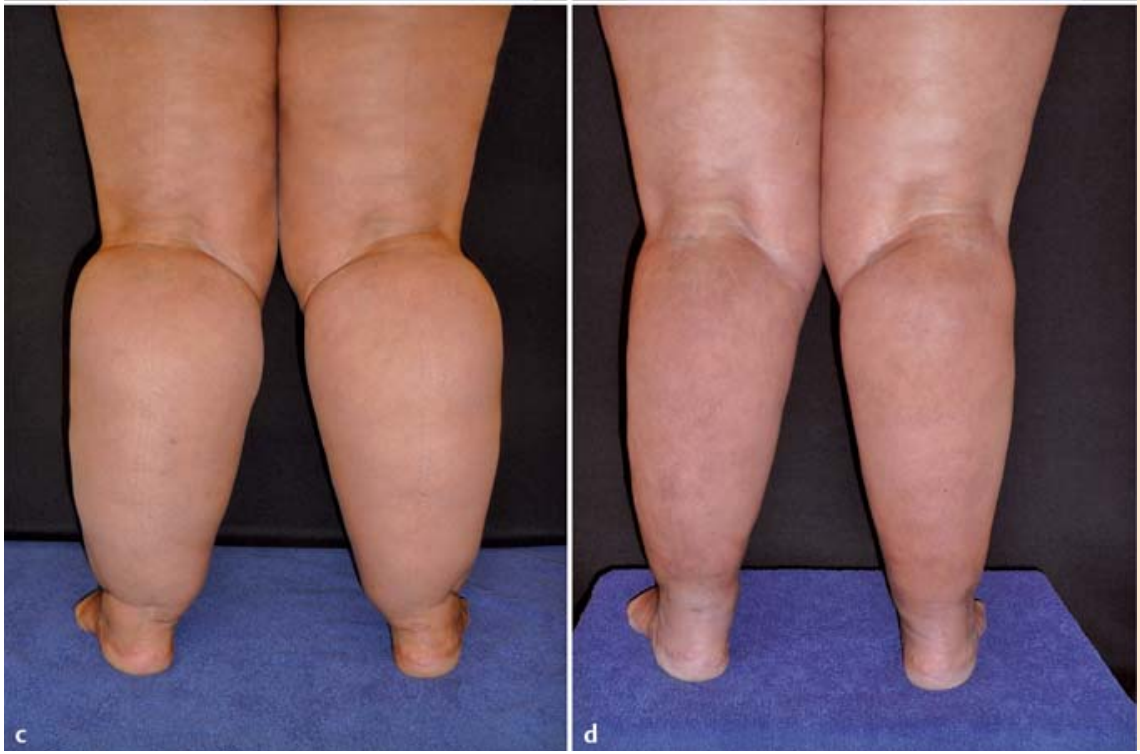

Für Patienten mit Analbuminämie ist zu beachten, dass Medikamente mit klinisch bedeutsamer Albuminbindung, wie insbesondere Herzglykoside, Phenprocoumon (Marcumar ${ }^{\mathrm{R}}$ ), Furosemid und Phenytoin, aber auch Diclofenac, Ibuprofen, Tolbutamid und Cotrimoxazol, mit Vorsicht eingenommen werden sollten. Zum einen kann eine Dosisreduktion sinnvoll sein, da aufgrund des höheren Anteils des freien Wirkstoffs im Plasma eine stärkere Wirkung vorliegen kann. Zum anderen kann sich aber auch durch die fehlende Albuminbindung die Eliminationshalbwertzeit verkürzen, sodass eine höhere Dosierung notwendig ist [14]. Daher werden teilweise einschleichende Dosierungen unter enger klinischer Beobachtung, u.U. mit Bestimmungen des Plasmaspiegels, empfohlen.

Wie groß bei Analbuminämie im Einzelfall das Risiko der Entwicklung eines Lipödems ist, ist ebenso unklar. Insgesamt weist nur ein kleiner Teil der betroffenen Frauen diese Erkrankung auf. Bei der Aufstellung von insgesamt 50 Fällen im „Register of
Analbuminemia Cases" fanden sich bei 22 Frauen 4 Betroffene (18\%) mit Lipödem [17]; werden unsere beiden Patientinnen mit einbezogen, liegt diese Zahl bei 25\%. Unter Berücksichtigung der Häufigkeit von stationär behandelten Lipödempatientinnen in deutschen lymphologischen Kliniken mit einer Größenordnung von 8 bis 17\% [19] kann dies - trotz fehlender epidemiologischer Studien zum Lipödem in der Normalbevölkerung - als eine überzufällige Häufung angesehen werden.

Bei den Lipödempatientinnen lässt sich durch das sichere Verfahren der Liposuktion in Tumeszenz-Lokalanästhesie [13] - meist in Kombination mit konservativer Behandlung - eine deutliche Verbesserung der Körperform mit Normalisierung der Gesamtproportionen erreichen; zusätzlich kommt es zur Verminderung bzw. zum Verschwinden der lipödem-typischen Beschwerden $[22,23]$.

2011 wurde erstmals über die operative Behandlung eines Patienten mit Analbuminämie anlässlich eines Bypasses bei 
koronarer Herzkrankheit berichtet [8]; dabei wurden präoperativ Albumininfusionen bis zum Erreichen normaler Plasmawerte gegeben. Über die Behandlung eines die Analbuminämie begleitenden Lipödems mittels Liposuktion existieren unseres Wissens bisher nur zwei Kasuistiken; in beiden Fällen wurden die Eingriffe in Vollnarkose durchgeführt $[12,18]$. Bei einer Patientin trat bei der ersten Liposuktion eine Poplitealvenenthrombose und beim zweiten Eingriff eine Phlegmone gluteal auf [12]. In der zweiten Kasuistik wurde über keine Komplikationen berichtet [18]. Sowohl in diesen beiden Fällen als auch bei unserem Geschwisterpaar erfolgte keine vorherige Albumingabe.

\section{Fazit für die Praxis \\ $\nabla$}

Prinzipiell sollte bei allen Patientinnen mit Lipödem und erhöhten Blutfettwerten (Hyperlipidämie) - insbesondere in Kombination mit einer erhöhten Blutsenkungsreaktion - an das sehr seltene Krankheitsbild der Analbuminämie gedacht und eine Serum-Protein-Elektrophorese durchgeführt werden. Während die Analbuminämie normalerweise kaum Probleme bereitet, kann das immer Beschwerden bereitende Lipödem operativ mittels Liposuktion in Tumeszenz-Lokalanästhesie erfolgreich behandelt werden.

\section{Interessenkonflikte \\ $\nabla$}

Die Autoren geben an, dass keine Interessenkonflikte bestehen.

\section{Abstract}

\section{Analbuminaemia in Combination with Lipoedema in Two Siblings}

We present two Turkish sisters with the rare disease congenital analbuminaemia in combination with lipoedema. Analbuminaemia, always associated with hyperlipidaemia and increased erythrocyte sedimentation rate (ESR), hardly causes any complaints and therefore often is diagnosed only by chance. In contrary lipoedema is characterized by an obvious phenotype with disproportionate body proportion due to an increased amount of subcutaneous fatty tissue in combination with oedema, pain due to pressure and haematomas.

While analbuminaemia itself needs no further intervention, lipoedema can be treated successfully by liposuction. The combination of both diseases should be known.

\section{Literatur}

1 Allen EU, Hines EA. Lipedema of the legs: A syndrom characterized by fat legs and orthostatic edema. Proc Staff Meet Mayo Clin 1940; 15: $184-187$

2 Bennhold H, Peters H, Roth E. Über einen Fall von kompletter Analbuminämie ohne wesentliche klinische Krankheitszeichen. Verh Dtsch Ges Inn Med 1954; 60: 630-634

3 Berger GM, Stephen CR, Finestone A et al. Analbuminaemia. Clinical and laboratory features in a South African patient. S Afr Med J 1985; 67: $418-422$

4 Burgess $L J$, Marais $A D$. The use of Simvastatin in analbuminaemia. Cardiovasc Drugs Ther 2001; 15: $555-558$

5 Campagna F, Fioretty $F$, Burattin $M$ et al. Congenital analbuminemia attributable to compound heterozygosity for novel mutations in the albumin gene. Clinical Chemistry 2005; 51: $1256-1258$

6 Child $A H$, Gordon $K D$, Sharpe $P$ et al. Lipedema: an inherited condition. Am J Med Genet A 2010; 152A: 970-976

7 Dagnino M, Caridi G, Marsciani $M$ et al. A novel frame-shift deletion causing analbuminaemia in an Italian paediatric patient. Eur J Clin Invest 2010; 40: $281-284$

8 Demirsoy E, Girin G, Ozker E. Coronary artery bypass in a patient with analbuminemia. Tex Heart Inst J 2011; 38: 85 -87

9 Fife CE, Maus EA, Carter MJ. Lipedema: A frequently misdiagnosed and misunderstood fatty deposition syndrome. Advances in Skin \& Wound Care 2010; 23: $81-94$

10 Figueira TR, Vercesi AE, Oliveira HCF. Lack of plasma albumin impairs intravascular lipolysis and explains associated free fatty acids deficiency and hypertriglyceridemia. Lipids Health Dis 2010; 9: 146-151

11 Galliano M, Campagnoli M, Rossi A et al. Molecular diagnosis of analbuminemia: A novel mutation identified in two Amerindian and two Turkish families. Clinical Chemistry 2002; 48: 844-849

12 Gössi B, Kleinert $U$, Gössi $U$. Ein weiterer Fall von Analbuminämie. Schweiz Med Wochenschr 2000; 130: 583-589

13 Habbema L. Safety of liposuction using exclusively tumescent local anesthesia in 3,240 consecutive cases. Dermatol Surg 2009; 35: 1728 1735

14 Inoue $M$, Okajima $\mathrm{K}$, Itoh $\mathrm{K}$ et al. Mechanism of furosemid resistance in analbuminemic rats and hypoalbuminemic patients. Kidney Int 1987; 32: $198-203$

15 Kallee E. Bennhold's analbuminaemia: a follow-up study of the first two cases (1953-1992). J Lab Clin Med 1996; 127: 470-480

16 Koot BG, Houwen R, Pot DJ et al. Congenital analbuminaemia: biochemical and clinical implications. A case report and literature review. Eur J Pediatr 2004; 163: 664-670

17 Kragh-Hansen $U$. Register of analbuminemia cases (database on the Internet). www.albumin.org

18 Kühn-Velten WN, Busch K, Reinhauer H et al. Progressive lipodystrophy in a patient with analbuminemia: laboratory investigation and treatment by ultrasound-assisted lipoplasty. Eur J Plast Surg 2001; 24: $197-200$

19 Meier-Vollrath I, Schneider W, Schmeller W. Lipödem: Verbesserte Lebensqualität durch Therapiekombination. Dtsch Ärzteblatt 2005; 102: A1061 - 1067

20 Nicholson JP, Wolmarans MR, Park RG. The role of albumin in critical illness. Br J Anaesth 2000; 85: 599-610

21 Rapprich S, Dingler A, Podda M. Liposuktion ist eine wirksame Therapie beim Lipödem - Ergebnisse einer Untersuchung mit 25 Patientinnen. JDDG 2011; 9: $33-40$

22 Schmeller W, Meier-Vollrath I. Lipödem - Neues zu einem weitgehend unbekannten Krankheitsbild. Akt Dermatol 2007; 33: 251 - 260

23 Schmeller $W$, Hüppe M, Meier-Vollrath I. Liposuction in lipoedema yields good long-term results. Brit J Dermatol 2012; 166: 161 - 168

24 Wienert $V$, Földi $E$, Jünger $M$ et al. Lipödem. Leitlinien der Deutschen Gesellschaft für Phlebologie. Phlebologie 2009; 38: 164-167 